\title{
Article
}

\section{DC modulation noise in clustered particulate media}

Parker, D.A., Kay, G.E., Bissell, P.R. and Mercer, Tim

Available at http://clok.uclan.ac.uk/10736/

Parker, D.A., Kay, G.E., Bissell, P.R. and Mercer, Tim ORCID: 0000-0002-15572138 (2002) DC modulation noise in clustered particulate media. Journal of Magnetism and Magnetic Materials, 242-24 . pp. 366-369. ISSN 03048853

It is advisable to refer to the publisher's version if you intend to cite from the work. http://dx.doi.org/10.1016/S0304-8853(01)01161-1

For more information about UCLan's research in this area go to http://www.uclan.ac.uk/researchgroups/ and search for < name of research Group>.

For information about Research generally at UCLan please go to http://www.uclan.ac.uk/research/

All outputs in CLoK are protected by Intellectual Property Rights law, including Copyright law. Copyright, IPR and Moral Rights for the works on this site are retained by the individual authors and/or other copyright owners. Terms and conditions for use of this material are defined in the policies page.

\section{CLoK}

Central Lancashire online Knowledge www.clok.uclan.ac.uk

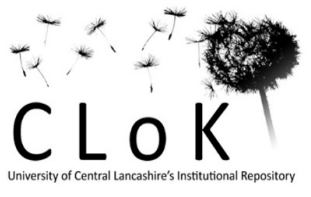


NOTICE: this is the author's version of a work that was accepted for publication in the Journal of Magnetism and Magnetic Materials. Changes resulting from the publishing process, such as peer review, editing, corrections, structural formatting, and other quality control mechanisms may not be reflected in this document. Changes may have been made to this work since it was submitted for publication. A definitive version was subsequently published in the J. Magn. Magn. Mater., Vol. 242, Part 1 (2002) p. 366 DOI: $\underline{10.1016 / S 0304-8853(01) 01161-1}$

\title{
DC modulation noise in clustered particulate media
}

\author{
D.A. Parker ${ }^{\mathrm{a}}$, G.E. Kay ${ }^{\mathrm{a}}$, P.R. Bissell ${ }^{\mathrm{a}}$, T. Mercer $^{\mathrm{a}}$ \\ ${ }^{\mathrm{a} C e n t r e ~ f o r ~ M a t e r i a l s ~ S c i e n c e, ~ U n i v e r s i t y ~ o f ~ C e n t r a l ~ L a n c a s h i r e, ~ P r e s t o n, ~ P R 1 ~ 2 H E, ~ U K ~}$
}

\begin{abstract}
Mallinson's theory of recording media noise is extended to ensembles where the particles have an easy axis distribution or are clustered. The effect of clusters is that there is a critical value of packing fraction at which noise in the demagnetised state switches to a minimum. Comparison is made with measurements of DC modulation noise in double layer MP tape.
\end{abstract}

Keywords: Statistical model, DC noise, Clusters, Particulate recording media

Corresponding Author: Dr D.A. Parker, Centre for Materials Science, University of Central Lancashire, PRESTON PR1 2HE, UK.

daparker@uclan.ac.uk, Tel: +44 - 1772 - 893550, Fax: +44 - 1772 - 892996 
Noise in magnetic recording media occurs because of statistical variations in the properties of an ensemble of magnetic particles. Previous investigations of noise have considered variations in the magnetisation of such an ensemble [1-3]. Mallinson [2] developed a model of noise in particulate media, which also included variations in the packing density of the particles. He considered identical particles to be aligned either parallel or anti-parallel to the field direction and this always generated a maximum total noise in the demagnetised state. In this paper we extend Mallinson's model to include (i) a distribution of easy axes and (ii) clustering of particles with a given distribution of cluster sizes.

Without clusters the total noise always has a maximum in the demagnetised state, which results from the increased number of arrangements of the particle moments in the ensemble in that state. The effect of including clusters is that, for each mean cluster size, there is a critical packing fraction below which the total noise in the demagnetised state switches from a maximum to a minimum.

Mallinson's model can be extended for separate particles by introducing a distribution of easy axes. Consider an assembly of $N$ particle sites with volume packing fraction $\bar{P}$. Let each particle have a magnetic moment of magnitude $\mu$ and orientation in three dimensions represented by angles $\alpha$ and $\beta$, where $\alpha$ is the angle between the in-plane component of the moment and the longitudinal direction and $\beta$ is the angle between the moment and the plane of the tape. The moment measured in the longitudinal direction of the tape is $\mu_{x}=\mu \bar{m} \cos \alpha \cos \beta$ where the normalised mean magnetisation $\bar{m}=2 p-1$ and $p$ is the probability that a moment is in the positive direction of its easy axis. Assuming that $f(\alpha, \beta)$ is the probability density function of easy axis directions total noise is given by

$$
\sigma_{m}^{2}=N\left\{E\left(\mu_{x}^{2}\right)-\left(E\left(\mu_{x}\right)\right)^{2}\right\}
$$


where

$$
\begin{aligned}
& E\left(\mu_{x}^{2}\right)= \\
& \bar{P} \mu^{2} \int_{-\pi / 2}^{\pi / 2} \int_{-\pi / 2}^{\pi / 2} f(\alpha, \beta) \cos ^{2} \alpha \cos ^{3} \beta d \alpha d \beta
\end{aligned}
$$

and

$$
\begin{aligned}
& E\left(\mu_{x}\right)= \\
& \bar{P} \mu \bar{m} \int_{-\pi / 2}^{\pi / 2} \int_{-\pi / 2}^{\pi / 2} f(\alpha, \beta) \cos \alpha \cos ^{2} \beta d \alpha d \beta .
\end{aligned}
$$

The results for a 2-D distribution are obtained by substituting $\alpha=\theta$ and $\beta=0$ in equations (2) and (3). Simulations have been carried out for a number of easy axis distributions in both two and three dimensions. In three dimensions, both random and a truncated bivariate distribution were used to generate easy axes. It was assumed that $\alpha$ and $\beta$ are independent variables. Other experimental measurements [4] on the MP tape of this study have shown that the in-plane and out-of-plane easy axis distributions possess standard deviations of approximately 0.5 and 0.25 radians respectively and these estimates are used here for the bivariate model.

The results highlight that total noise is dependent on the packing fraction (Fig. 1). At the demagnetised states noise reduces as the packing fraction decreases; this was consistent for all distributions of easy axes. The packing fraction influences the degree of variation in the noise between the demagnetised and saturated states. When the packing fraction is large the total noise reduces rapidly as the magnetisation approaches saturation, whereas reducing the packing fraction flattens the noise distribution. There is a critical value of the packing fraction for which total noise at the saturated states is a maximum. When the easy axes are distributed randomly then the total noise, at both saturated and demagnetised states, is less than that generated from the bivariate distribution. Maximum 
noise occurs in the demagnetised state when the easy axes are orientated in a narrow region, the limiting case of this being Mallinson’s model.

In order to introduce particle clusters into the system, consider now that the ensemble consists of $N$ sites at each of which could be a cluster of $k$ particles. $\bar{P}$ is the probability that a cluster is present at a particular site. Let $\chi_{k}$ denote the probability that a non-empty site contains a cluster of size $k$. Let $X$ be a random variable representing the moment of a cluster in the longitudinal direction. For a cluster of size $k, X$ may take the values $(k-2 r) \mu_{x}, \quad r=0, \ldots, k$, with probabilities $\left(\begin{array}{l}k \\ r\end{array}\right) p^{k-r}(1-p)^{r}$. Therefore, the contribution to total noise is

$$
\sigma_{m}^{2}=N\left\{E\left(X^{2}\right)-(E(X))^{2}\right\}
$$

where

$$
E(X)=\bar{P} \bar{m} E\left(\mu_{x}\right) \sum_{k=1}^{\infty} k \chi_{k}
$$

and

$$
E\left(X^{2}\right)=\bar{P} E\left(\mu_{x}^{2}\right) \sum_{k=1}^{\infty} k\left\{(k-1) \bar{m}^{2}+1\right\} \chi_{k}
$$

If $K$ is a random variable representing cluster size then

$$
E(X)=\bar{P} \bar{m} E\left(\mu_{x}\right) E(K)
$$

and

$$
E\left(X^{2}\right)=\bar{P} E\left(\mu_{x}^{2}\right)\left\{\bar{m}^{2} E\left(K^{2}\right)+\left(1-\bar{m}^{2}\right) E(K)\right\}
$$

A truncated Poisson distribution has been used to generate the cluster size. Hence, the probability of obtaining a cluster size $k$ is given by $\chi_{k}=e^{-\lambda} \lambda^{k} / k !\left(1-e^{-\lambda}\right), k=1,2,3 \ldots$ and the expectations for this distribution are given by $E(K)=\lambda /\left(1-e^{-\lambda}\right)$ and $E\left(K^{2}\right)=\left(\lambda+\lambda^{2}\right) /\left(1-e^{-\lambda}\right)$. 
Introducing clusters has a significant impact on the noise distribution. When clusters are present at a site, reducing the packing fraction transforms the noise distribution from a maximum to a minimum at the demagnetised states. This is demonstrated in Fig. 2 where the expected cluster size per site is fixed and the easy axes are generated from the bivariate distribution. Similar dependence on packing fraction has been reported for particulate rigid disks [5]. As the packing fraction decreases then the noise at the demagnetised states is reduced, this is consistent with systems of single particles.

The effect of increasing the mean cluster size is demonstrated for the bivariate case in Fig. 3 when $\bar{P}=0.5$. As the mean cluster size increases then noise becomes more pronounced at the saturated states. It was also observed that for a clustered system the noise distribution was a maximum only when the packing fraction was sufficiently large. When the packing fraction was large an increase in $\lambda$ produced less variability between the demagnetised and saturated states until eventually a critical value of $\lambda$ transformed the distribution from a maximum to a minimum.

The statistical model described has been compared with a typical experimental result on a double layer MP tape (single magnetic layer). The volume packing fraction of $13 \%$ for the magnetic material only (i.e. excluding the particle passivating layer) was estimated from bulk magnetic measurements and given values for the type of particle used in this media (Fig. 4). It should be noted that this seemingly very low value corresponds to $\sim 30$ $\%$ for the actual particles if the passivating (non-magnetic) particle shell is included.

The method used to generate the noise curve modulates the DC state in the same manner as that commonly used for DCD remanence investigations (eg [6]). For this, the sample is initially conditioned using an in-plane (say positive direction) saturating field. A reverse field in the opposing (negative) direction is then applied in a series of increasing steps. Within each step the reverse field is reduced to zero to allow the remanent state to be measured before the next field step is applied. 
The noise power at each remanent state of figure 4 was found by passing the tape over a compatible read-head at a speed of $0.5 \mathrm{~ms}^{-1}$. The subsequent voltage-time signal from the head was captured at a rate of $250 \times 10^{6}$ samples per second by a digital storage oscilloscope (DSO) and the total noise power determined using the DSO signal processing software. The magnetisation values of the abscissa (normalised to the remanent saturated state $M_{r}$ ) were determined from conventional DCD plots of $M$ as a function of reverse field. The resultant curve shows a broad 'valley' with the minimum centred about the demagnetised state. There is general qualitative agreement with the model for a system of clustered particles. It is also consistent with previous experimental studies on other particulate tapes that show a trough, or noise minimum, near to the remanent coercivity, $H_{r}[7,8]$. This is typically interpreted in terms of flux closure effects due to the clustering of particles that is expected in real systems, which reduces the stray flux emanating from these clusters in the demagnetised state as 'seen' by the read-head.

Although this model gives a qualitative agreement with the result in figure 4, there are small asymmetric features in the experimental results which cannot be matched by a simple statistical model. Furthermore, there are other examples where the asymmetry is exaggerated $[8,9]$. This must result from the complex magnetisation states generated in magnetic media as they are subject to applied magnetic fields. However, the model does extend the approach of Mallinson to give a more realistic representation but still maintains a simple physical interpretation of the features.

We can conclude that the statistical noise model, which takes into account various easy axis distributions for both systems of single particles and clusters of particles has highlighted that the total noise distribution is dependent on a subtle relationship between the packing density and the mean cluster size in the system. The clustered model compares favourably with experiment and provides the basis for further developments. 


\section{Acknowledgements}

We are grateful to the National Storage Industries Consortium (NSIC) and the Engineering and Physical Sciences Research Council (EPSRC), grant GR/N64069/01, for financial support for this project on particulate media noise.

\section{References}

[1] L. Thurlings, IEEE Trans. Magn. 16 (1980) 507.

[2] J.C. Mallinson, IEEE Trans. Magn. 27 (1991) 3519.

[3] T.J. Silva, H.N. Bertram, IEEE Trans. Magn. 26 (1990) 3129.

[4] M. Vopsariu, P.R. Bissell, R. Cookson, Presented at SCM2001 conference, Seeheim, September 2001 and submitted to Phys. Stat. Solid.

[5] N. Kodama, J. Magn. Magn. Mater. 224 (2001) 113.

[6] R.W. Chantrell, K. O’Grady, J. Phys. D: Appl. Phys. 25 (1992) 1.

[7] M.D. Clarke, P.R. Bissell, R.W. Chantrell, R.G. Gilson, J. Magn. Magn. Mater. 95 (1991) 17.

[8] S.M. McCann, P.R. Bissell, T. Onions, T. Mercer, J. Magn. Magn. Mater. 183 (1998) 220.

[9] P.R. Bissell, T. Mercer, P. Ardeleanu, A. Stancu, L. Stoleriu, Paper HF-03, MMM’01 conference, Seattle, November 2001 and submitted to J. Appl. Phys. 


\section{Figure captions}

Fig. 1. Noise distribution for various $\bar{P}$, for an unclustered, system, when the easy axes are generated from a bivariate distribution

Fig. 2. Noise distribution for various $\bar{P}$, for a clustered system, when the easy axes are generated from a bivariate distribution. The expected cluster size per site is fixed at $\lambda=3$.

Fig 3. Noise distribution for various $\lambda$ when $\bar{P}=0.5$ and the easy axes are generated from a bivariate distribution.

Fig. 4. Experimental DC modulation noise measurements on particulate tape. The broad minimum about the demagnetised state is best described by the clustered model. 
Figure 1, D.A. Parker et al.

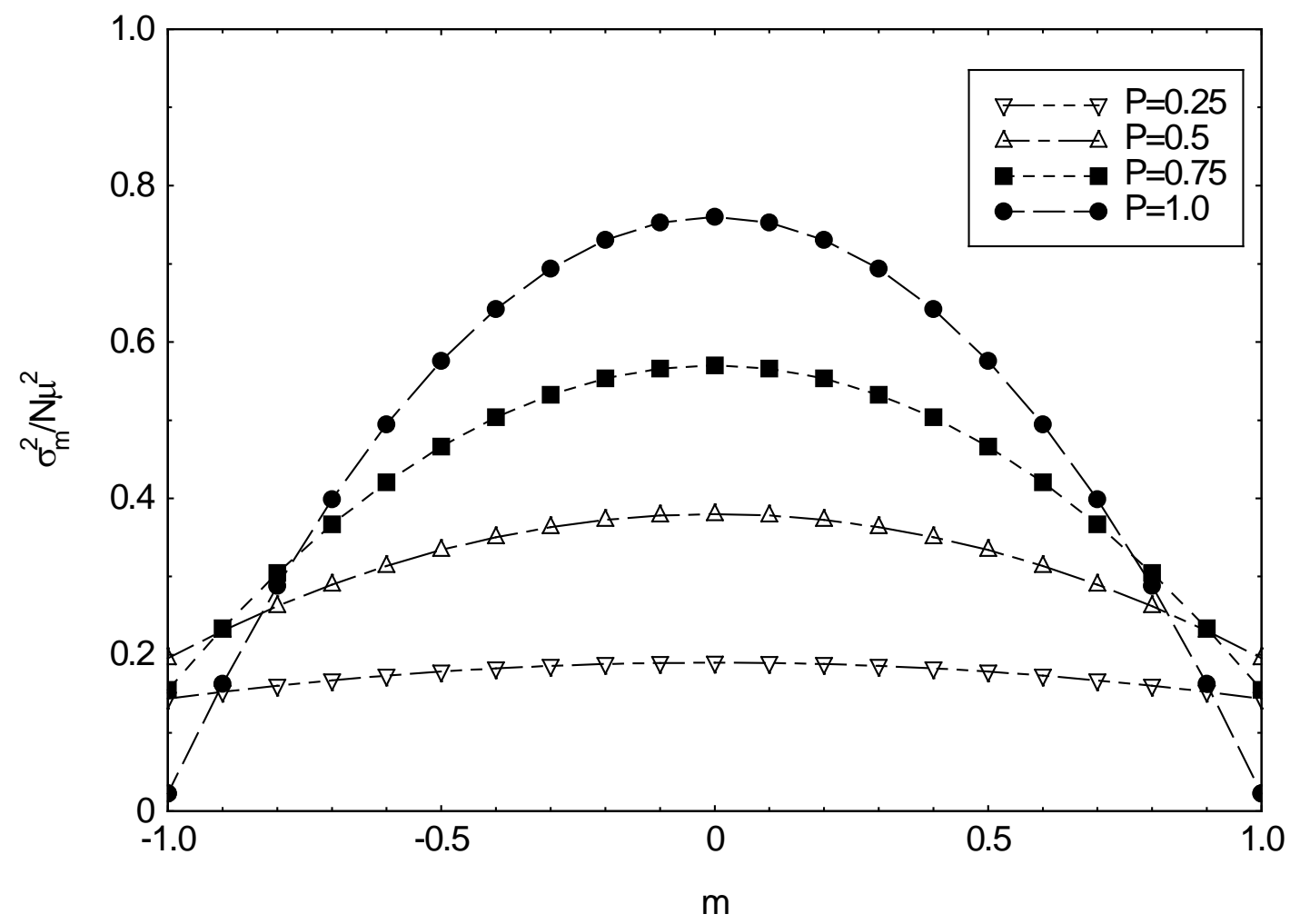

Suggested reduction factor $=0.5$

Figure 2, D.A. Parker et al.

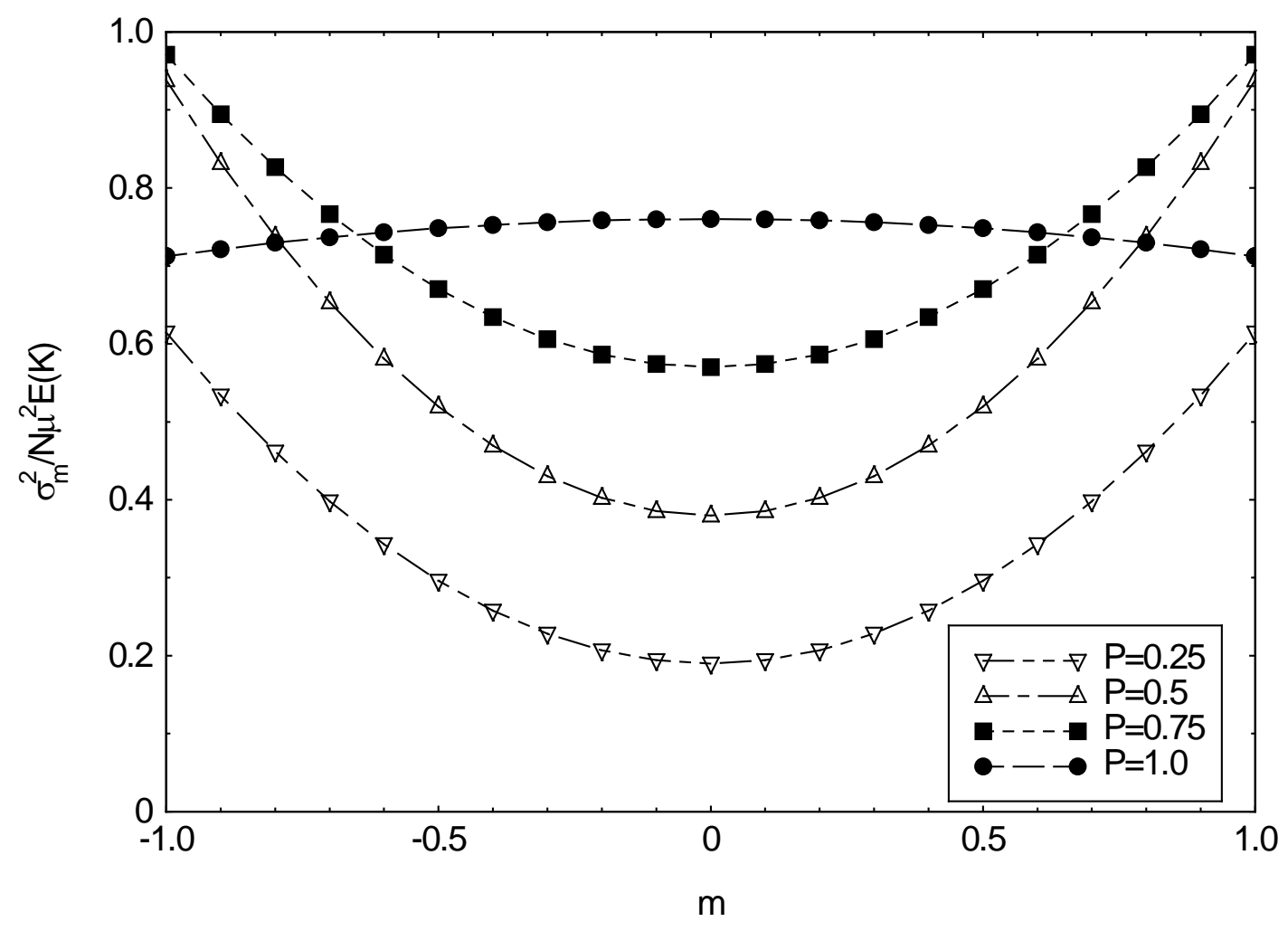

Suggested reduction factor $=0.5$ 
Figure 3, D.A. Parker et al.

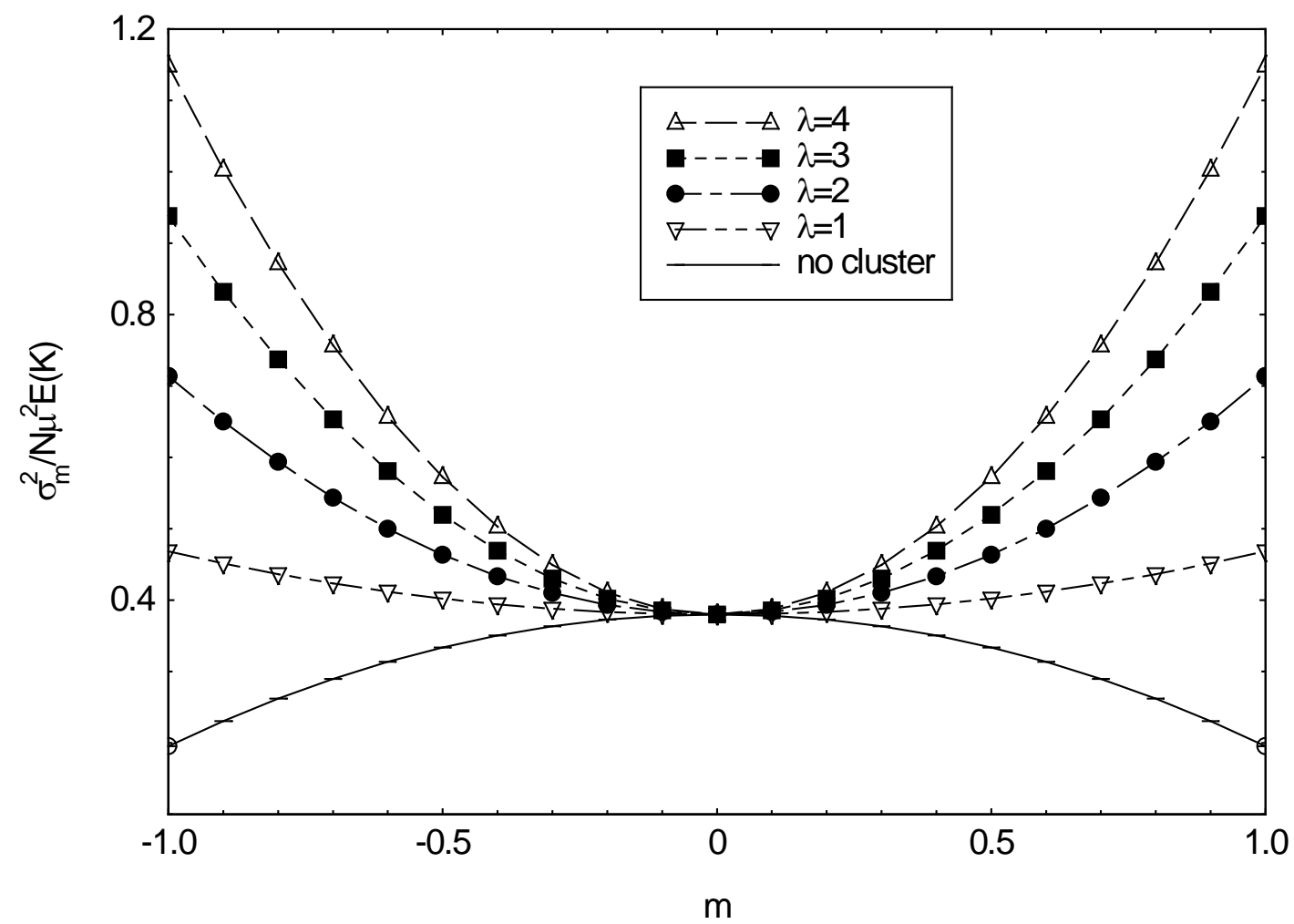

Suggested reduction factor $=0.5$

Figure 4, D.A. Parker et al.

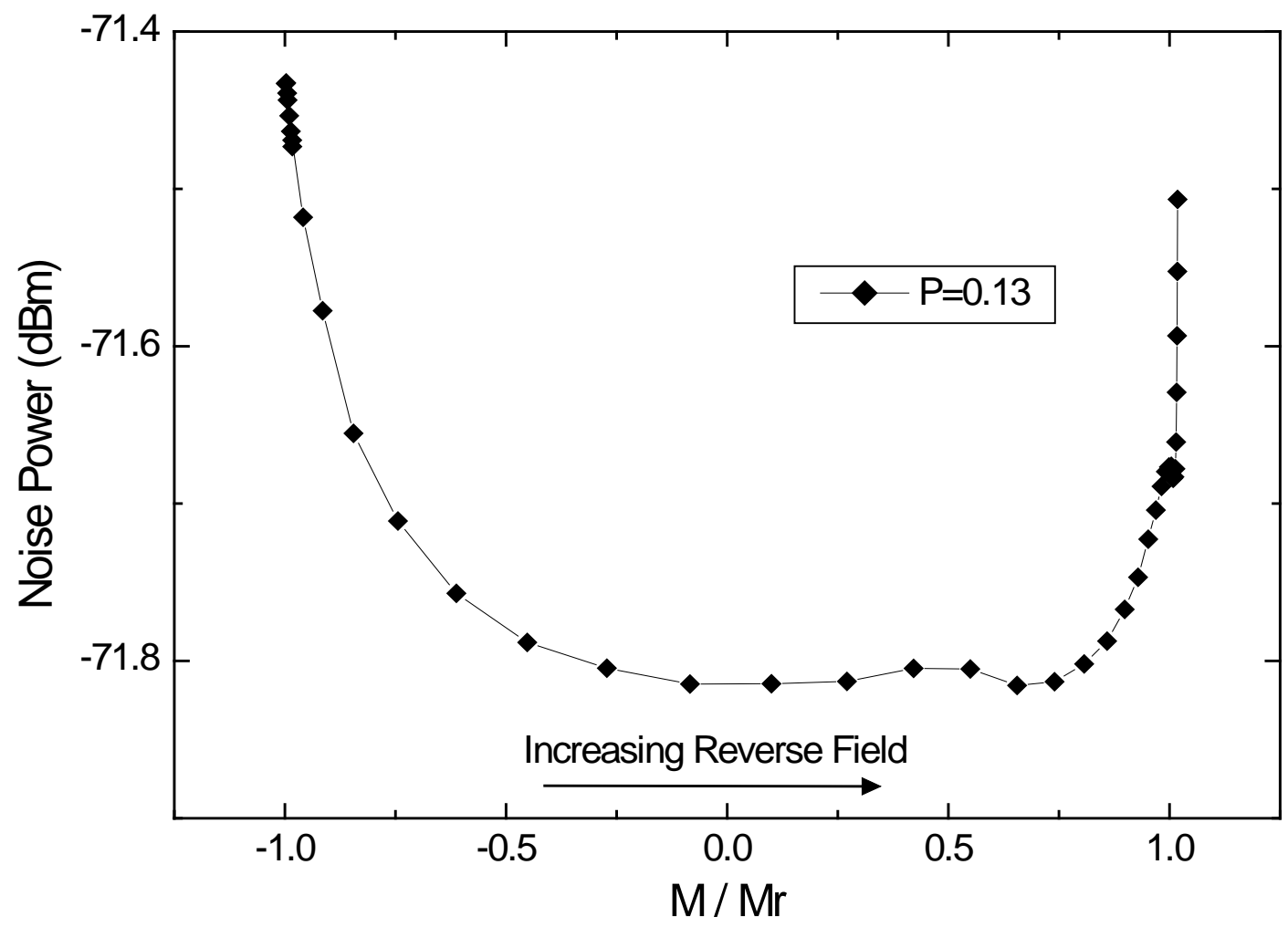

Suggested reduction factor $=0.5$ 